\title{
4 DIE ROLLE VON SCHLÜSSELPERSONEN FÜR SOZIALRÄUMLICHEN WANDEL - EIN FALLbEISPIEL AUS ÖSTERREICH
}

Stefanie DörINGER, Institut für Stadt- und Regionalforschung, Österreichische Akademie der Wissenschaften

\section{INHALT}

4.1 Einleitung

4.2 Theoretischer Hintergrund

4.2.1 Lokale und regionale Governance: eine analytische Perspektive.......................51

4.2.2 Institutional, Policy und Governance Entrepreneurship ……….......................52

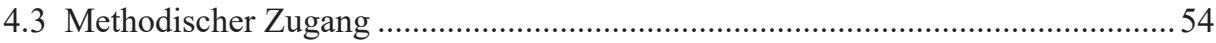

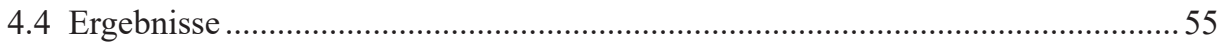

4.4.1 Case Study Waidhofen/Ybbs und der Beta-Campus.........................................55

4.4.2 Lokale Governance-Strukturen und ein Unternehmer als Schlüsselperson........56

4.4.3 Der Unternehmer und die verschiedenen Aspekte sozialräumlichen Wandels ...56

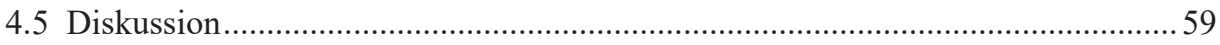

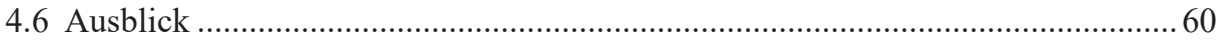

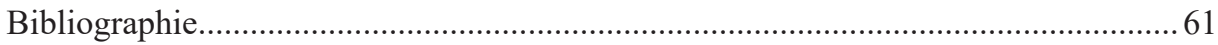

Vor dem Hintergrund von sozialen und ökonomischen Transformationsprozessen in ländlichen Räumen, wird in Wissenschaft und Praxis zunehmend die zentrale Rolle von Schlüsselpersonen aus Politik, Wirtschaft oder Zivilgesellschaft für sozialräumliche Wandlungsprozesse betont. Der vorliegende empirische Beitrag legt das Hauptaugenmerk auf einen Unternehmer, der mit der Entwicklung eines Innovationshubs eine Schlüsselfunktion in der regionalen Wirtschaftsentwicklung einnimmt. Vor 
dem Hintergrund von Governance- und Entrepreneurship-Konzepten wird herausgearbeitet, was den Unternehmer auszeichnet und welchen Einfluss er auf sozialräumliche Wandlungsprozesse nimmt. Zielsetzung ist es, den Handlungsspielraum von Schlüsselpersonen anhand einer differenzierten Betrachtung von sozialräumlichen Wandlungsprozessen exemplarisch für eine Region zu skizzieren. Anhand des Fallbeispiels wird deutlich, dass das persönliche Netzwerk eine zentrale Handlungsressource für Schlüsselpersonen darstellen kann, um sozialräumliche Wandlungsprozesse zu initiieren. Dabei zeigt sich, dass sozialräumlicher Wandel auch mit Veränderungen des regionalen Steuerungs- und Entscheidungsgefüge einhergehen kann.

\subsection{Einleitung}

Die Verschärfung räumlicher Disparitäten durch demographische und ökonomische Entwicklungen, wie Abwanderung und Strukturwandel, stellt ländliche Räume und ihre Akteure vor komplexe Herausforderungen. In diesem Zusammenhang wird in Wissenschaft und Praxis häufig die zentrale Rolle von Schlüsselpersonen aus Politik, Wirtschaft oder Zivilgesellschaft für sozialräumliche Wandlungsprozesse betont. Schlüsselpersonen engagieren sich auf außergewöhnliche Weise für die Entwicklung einer Region, verändern räumliche Praktiken oder beeinflussen maßgeblich Wandlungsprozesse. Aus geographischer und planerischer Sicht ist jedoch bisher weitgehend unklar, über welchen Gestaltungsspielraum Einzelpersonen im Kontext von sozialräumlichen Wandlungsprozessen tatsächlich verfügen (GAILING \& IBERT 2016) und inwieweit übergeordnete Ebenen und institutionelle Faktoren ihr Handeln beeinflussen.

Der vorliegende Beitrag greift diese Thematik auf und legt das Hauptaugenmerk auf einen Unternehmer, der sich aktiv in die regionale Wirtschaftsentwicklung einbringt und auf ein umfangreiches Netzwerk an Unterstützerinnen zurückgreifen kann. Das Handeln des Unternehmers hat dabei nicht nur Einfluss auf die wirtschaftliche Ausrichtung der Region, sondern verändert auch das lokale Steuerungs- und Entscheidungsgefüge. Zielsetzung ist es, vor dem Hintergrund von Governance- und EntrepreneurshipKonzepten, den Beitrag des Unternehmers zu sozialräumlichen Wandlungsprozessen exemplarisch zu analysieren. Berücksichtigung finden dabei auch übergeordnete sozioökonomische Entwicklungen und institutionelle Rahmenbedingungen. Von Interesse sind folgende Fragen: Was zeichnet die Schlüsselperson aus, über welche Handlungsressourcen verfügt sie und welche Zielsetzungen verfolgt sie? Welche Rolle nimmt sie im regionalen Entscheidungs- und Steuerungsgefüge ein und inwieweit beeinflusst sie sozialräumliche Wandlungsprozesse? 


\subsection{Theoretischer Hintergrund}

\subsubsection{Lokale und regionale Governance: eine analytische Perspektive}

Um zu verstehen, ob und inwiefern Schlüsselpersonen sozialräumliche Entwicklungsprozesse auf lokaler bzw. regionaler Ebene beeinflussen können, kann die analytische Perspektive auf lokale und regionale Governance-Strukturen einen zentralen Beitrag leisten. Diese Perspektive ermöglicht es die Einbettung von Schlüsselpersonen in öffentliche und politische Steuerungs- und Entscheidungsstrukturen, in die eine Vielzahl von staatlichen und nicht-staatlichen Akteuren eingebunden sind, besser zu verstehen. Auch wenn es keine einheitliche Definition des Governance-Begriffs gibt, so können wesentliche Merkmale des theoretischen Konzepts identifiziert werden. BeNZ et al. (2008) definiert Governance als „Steuern und Koordinieren mit dem Ziel des Managements von Interdependenzen zwischen Akteuren." Diese komplexen Steuerungs- und Koordinationsstrukturen können hierarchische, kompetitive und kooperative Akteursbeziehungen umfassen. Die handelnden Akteure werden von formellen und informellen Regelwerken, wie Gesetzen, Richtlinien oder Normen, beeinflusst. Die Akteure selbst zeichnen sich durch spezifische Handlungsorientierungen und Handlungsressourcen aus. Erstere werden wesentlich von individuellen Vorstellungen und Werten bestimmt, die wiederum auch die Präferenzen und Wahrnehmungen der Akteure definieren. Handlungsressourcen hingegen umfassen soziale, finanzielle oder auch technische Ressourcen, die die Akteure bei ihren Zielsetzungen unterstützen, aber auch einschränken können.

Obwohl in der Governance-Forschung vorrangig korporative Akteure, wie Körperschaften, Organisationen oder Vereine, im Mittelpunkt des Interesses stehen, können individuelle Akteure bzw. Schlüsselpersonen zentrale Rollen im Governance-Gefüge einnehmen (SCHARPF 2000, Cocks 2013). Gerade in der lokalen bzw. regionalen Governance kommt einzelnen Personen mitunter eine wichtige Position zu. Erstens kann davon ausgegangen werden, dass es sich auf lokaler Ebene um eine vergleichsweise überschaubare Anzahl an Akteuren handelt, die einander persönlich kennen und deren Interaktionen auf Vertrauen und sozialer Nähe aufbauen. Zweitens sind lokale Steuerungsstrukturen in Mehrebenen-Strukturen eingebettet, die sowohl staatliche, als auch nicht-staatliche Akteure umfassen und im Rahmen derer Schlüsselpersonen womöglich flexibler und schneller agieren können als Kollektive oder formale Zusammenschlüsse. Drittens können Schlüsselpersonen mit lokalem Wissen ihren Anliegen und ihren Interessen eher Gehör verschaffen und Ideen über persönliche Netzwerke durchsetzungsfähig machen (MAYER \& BAUMGARTNER 2014, BEer 2014). In Summe ist über die Interaktionen und den Einfluss von Schlüsselpersonen auf Prozesse der Regionalentwicklung bisher jedoch nur wenig bekannt (WILLI et al. 2018). Um sich konzeptionell dem Handlungsspielraum von Schlüsselpersonen zu nähern und der Vielfalt von sozialräumlichen 
Wandlungsprozessen zu nähern, werden nachfolgend die Konzepte Institutional, Policy und Governance Entrepreneurship vorgestellt.

\subsubsection{Institutional, Policy und Governance Entrepreneurship}

Der Begriff Entrepreneurship stammt ursprünglich aus den Wirtschaftswissenschaften und meint im weitesten Sinne innovatives Unternehmertum (SCHUMPETER 1961). Die politik- und organisationswissenschaftliche Perspektive hingegen, die in diesem Beitrag im Vordergrund steht, betont die Rolle von „Menschen mit Unternehmergeist" und ihre Bedeutung für gesellschaftliche Entwicklungsprozesse. Hervorgehoben werden dabei insbesondere ihre individuellen Charakteristika, ihre Fähigkeiten und Strategien. Nachfolgend wird zwischen den Konzepten des Institutional Entrepreneurship (Battilana et al. 2009), Policy Entrepreneurship (Mintrom \& Norman 2009) und Governance Entrepreneurship (DöRINGER 2020) unterschieden. Die konzeptionelle Unterscheidung in Institutional, Policy und Governance Entrepreneurship ermöglicht eine prozesshafte und differenzierte Perspektive auf das Handeln von Schlüsselpersonen in der Regionalentwicklung. Diese Konzepte erweisen sich für die Analyse sozialräumlicher Wandlungsprozesse insofern als besonders vielversprechend, da sowohl die individuellen Eigenschaften und Beziehungsgefüge von Personen als auch das kontextuelle Umfeld in den Blick genommen wird. Zentral ist dabei das Spannungsverhältnis von strukturellen Kontextbedingungen und dem individuellen Handlungsspielraum (GAILING \& IBERT 2016, Galanti 2018).

Policy Entrepreneurship geht auf das Multiple Streams Model von KIngdon (1995) zurück und fokussiert auf Policy Change (Mintrom \& Norman 2009), das heißt auf Wandlungsprozesse, die beispielsweise die Einführung von neuen politischen Maßnahmen oder Instrumenten umfassen. Dabei existieren nach KINGDON (1995) die drei Ströme „problems“, ,policies“ und „politics“. Diese treffen zu bestimmten Zeitpunkten zusammen und ermöglichen "windows of opportunities", die dann von Policy Entrepreneuren wahrgenommen werden. Policy Entrepreneure werden allgemein als Akteure definiert "who are willing to invest their resources - time, energy, reputation, money - to promote a position in return for anticipated future gain" (ibid., p. 179).

Der Begriff des Institutional Entrepreneurs hingegen bezieht sich auf "change agents who initiate divergent changes [...] that break the institutional status quo in a field of activity" (BATTILANA et al. 2009, p. 67). Sie zielen darauf ab, formelle oder informelle Institutionen zu verändern bzw. aufzubrechen. Während unter formellen Institutionen rechtlich bindende Regelwerke, wie z.B. Gesetze oder Verordnungen (NoRTH 1990), verstanden werden, umfassen informelle Institutionen allgemein anerkannte Verhaltensregeln, soziale Praktiken oder Normen.

Als dritte Kategorie neben Policy und Institutional Entrepreneurship wird in jüngerer Vergangenheit zunehmend Governance Entrepreneurship (PATTBERG 2017, WILLI et al. 2018, DöRINGER 2020) diskutiert. Das Konzept Governance Entrepreneur- 
ship nimmt die Veränderungen von regionalen Steuerungs- und Handlungsgefügen in den Blick (DöRINGER 2020). Dieser Wandel kann, muss aber von Einzelnen nicht bewusst intendiert sein. Veränderte Governance-Arrangements können beispielsweise ein Ergebnis angestrebter politischer oder institutioneller Veränderungen sein. Gleichzeitig können Schlüsselpersonen auch einen Wandel der lokalen oder regionalen GovernanceStrukturen beabsichtigen, um Einfluss in bestimmten Handlungsfeldern zu erlangen oder eine strategisch günstige Position in Entscheidungsprozessen zu erhalten. Obgleich sich diese Prozesse eher subtil und weniger öffentlichkeitswirksam als politische oder institutionelle Wandlungsprozesse präsentieren, sind sie als essentieller Faktor für sozialräumlichen Wandel zu bewerten, da grundlegende sozialräumliche Veränderungen auch mit einem Wandel von Steuerungs- und Handlungsgefügen einhergehen, um neue Maßnahmen entsprechend zu implementieren und Entscheidungen durchzusetzen. Zur weiteren analytischen Bestimmung lassen sich drei Dimensionen unterscheiden, die Veränderung durch Governance Entrepreneure erfahren können: Akteursgefüge, Interaktionsstrukturen und Handlungsräume.

Erstens kann Governance Entrepreneurship eine veränderte Zusammensetzung der handelnden Akteure im lokalen Steuerungsgefüge beinhalten, die sowohl mit einer Inklusion als auch Exklusion von Akteuren und Akteursgruppen einhergehen kann. Dabei können sich z.B. neue Partnerschaften zwischen Politik und Wirtschaft entwickeln, bei deren Initiierung und Gestaltung einzelne Personen zentrale Rollen einnehmen. Neben dieser horizontalen Erweiterung des Akteursspektrums können auf Basis von persönlichen Beziehungen und Netzwerken auch neue vertikale Achsen entstehen und Akteure aus übergeordneten politischen Ebenen in Entscheidungsprozesse eingebunden werden. Governance Entrepreneure können hier eine vermittelnde Schlüsselrolle besitzen und Funktionen eines Brokers übernehmen (Christopoulos \& InGOLD 2011, LeICK \& GRETZINGER 2018). Andererseits können Governance Entrepreneure auch einen Ausschluss bestimmter Akteure und Akteursgruppen anstreben, wenn Zielsetzungen nicht geteilt werden oder Konflikte im Gefüge zu Tage treten.

Zweitens können Governance Entrepreneure zu einer Veränderung von Interaktionsmodi beitragen. So können Governance Entrepreneure durch wiederkehrende dialogorientierte Handlungsmuster und Interaktionen beispielsweise dazu beitragen, dass sich vormals hierarchisch strukturierte Steuerungsgefüge zu einem kooperativ agierenden und dialogorientierten Netzwerk wandeln. Dies kann kurzfristig zu projektbezogenen Veränderungen führen, aber auch eine dauerhafte Umstrukturierung lokaler Entscheidungsstrukturen zur Folge haben. Auch ein veränderter Formalisierungsgrad von Interaktionen, etwa in Form von Verbänden oder Vereinen, kann ein Ergebnis von Governance Entrepreneurship sein (СROUCH 2005).

Drittens kann die Etablierung neuer Steuerungsgefüge und -netzwerke auch ein Re-Scaling von Handlungsräumen zur Folge haben. Die Etablierung von neuen Governance-Strukturen und Netzwerken kann mit einem veränderten räumlichen Bezugsrahmen der Steuerung einhergehen, der sich u.a. in Form von Regional Governance 
ausdrücken kann. Der Aufbau von regionalen Steuerungsstrukturen ermöglicht es Kommunen in ländlichen Gebieten, Ressourcen jenseits von administrativen Grenzen zu bündeln und somit den kommunalen Handlungsspielraum zu erweitern.

Ausgehend von unterschiedlichen theoretischen Zugängen konstituieren die drei vorgestellten Entrepreneurship-Konzepte verschiedene Rollen die Schlüsselpersonen in Wandlungsprozessen zeitgleich oder aufeinanderfolgend einnehmen können (siehe Tabelle 1). Diese drei Konzepte stehen in engem Zusammenhang und ihre Auswirkungen sind in der Praxis nicht immer trennscharf voneinander zu unterscheiden.

\section{Tabelle 1: Übersicht zu Policy, Institutional und Governance Entrepreneurship}

Policy Entrepreneurship Einführung oder Veränderung von (neuen) Policy-Inhalten und Maßnahmen, „policy change" (KINGDON 1995)
Etablierung oder Veränderung von formellen und informellen Institutionen, ,institutional change“" (BATTILANA et al. 2009, WeIK 2011)

\section{Governance Entrepreneurship}

Veränderung von Akteurskonstellationen, Interaktionsmodi und Handlungsräumen, „governance change“ (DöRINGER 2020)

\subsection{Methodischer Zugang}

Nachfolgend wird die Fallstudie der niederösterreichischen Kleinstadt Waidhofen/ Ybbs vorgestellt, die basierend auf einer Clusteranalyse und einer Medienanalyse ausgewählt wurde. Zunächst wurden explorative ExpertInneninterviews auf Landes- und Regionsebene geführt, um einerseits eine externe Expertise und allgemeine Einschätzungen über den individuellen Handlungsspielraum von Akteuren auf lokaler Ebene zu erhalten und um andererseits einen Überblick über aktuelle Projekte und das lokale Akteursgefüge in Waidhofen/Ybbs zu bekommen. Zudem wurde eine Dokumentenanalyse der zentralen Strategiepapiere und Entwicklungskonzepte der Stadtgemeinde durchgeführt.

Im Anschluss wurden qualitative Interviews mit Akteuren aus Politik, Wirtschaft und Verwaltung auf lokaler bzw. regionaler Ebene geführt (Schneeballverfahren). Dabei stellt die Identifikation von Schlüsselpersonen in der Praxis eine gewisse Herausforderung dar, da nicht alle Personen, die in Wandungsprozesse eingebunden sind, zwangsläufig eine Schlüsselfunktion einnehmen (SotARAUTA und PULKKINEN 2011, p. 100). Die 
Eigen- bzw. Fremdzuschreibung von Schlüsselpersonen in Form von Narrativen in den Interviews kann dabei lediglich als ein erster Hinweis dienen. Die eigentliche Zuordnung erfolgt über die konzeptionelle Abgrenzung von Schlüsselpersonen im Rahmen der vorgestellten Entrepreneurship Konzepte.

Entrepreneure sind dabei sowohl Forschungsgegenstand als auch potenzielle InterviewpartnerInnen. So gilt es bei der Erforschung von Schlüsselpersonen auf der einen Seite den ExpertInnenstatus zu bedenken, auf der anderen Seite aber auch die subjektiven Wahrnehmungen und Orientierungen der Befragten zu berücksichtigen. Zu diesem Zweck wurde ein methodischer Zugang entwickelt, der den Ansatz der theoriegenerierenden ExpertInneninterviews nach BoGNER \& MENZ (2009) und das problemzentrierte Interview nach WiTZEL (2000) kombiniert. Dieser problemzentrierte Zugang zu ExpertInneninterviews umfasst dabei sowohl narrative Elemente als auch spezifische Fragetechniken, um sich der subjektiven Dimension des ExpertInnenwissens und damit den Handlungsorientierungen und Wahrnehmungen der Schlüsselpersonen anzunähern.

\subsection{Ergebnisse}

\subsubsection{Case Study Waidhofen/Ybbs und der Beta-Campus}

Waidhofen/Ybbs ist eine Kleinstadt in Niederösterreich in voralpiner, ländlicher Lage. Die Statutarstadt hat rund 11.000 EinwohnerInnen und weist eine leicht rückläufige bis stagnierende Bevölkerungsentwicklung auf (STATISTIK Austria 2019). In der Vergangenheit hatte die Kleinstadt an der Eisenstraße mit einer Leerstandsproblematik in der Innenstadt zu kämpfen, die mit einem umfassenden Prozess zur Innenstadtbelebung bewältigt werden konnte. Waidhofen/Ybbs blickt auf eine lange Industrietradition in der Eisenproduktion zurück und die wirtschaftliche Struktur in der Region setzt sich aus größeren Leitbetrieben, aber auch vielen klein- und mittelständischen Unternehmen (KMUs) zusammen. Neben dem demographischen Wandel ist es vor allem der Fachkräftemangel, der die Region und ihre Akteure seit Jahren beschäftigt. Diese Herausforderungen werden in regionalen Entwicklungsstrategien berücksichtigt und im Rahmen verschiedener Initiativen aufgegriffen.

In den vergangenen Jahren wurde von Unternehmen aus der Region die Idee eines regionalen Innovationshubs entwickelt. Erster Schritt für dieses Projekt war eine Machbarkeitsstudie „Zukunfts-Campus Metal Highway“ im Rahmen eines LEADERProjekts im Jahr 2016. Die Realisierung des Technologie- und Innovationszentrum mit dem Namen Beta-Campus ist in den kommenden Jahren in einem leerstehenden Industrieareal in einem Ortsteil unweit des Zentrums von Waidhofen/Ybbs auf ca. 9.000 $\mathrm{m}^{2}$ geplant. In der Startphase wurde im Jahr 2018 bereits ein Co-Working Space mit entsprechenden Räumlichkeiten eingerichtet. In den kommenden Jahren sollen ein 
Ausbildungszentrum, Labore und Werkstätten für die heimischen Industriebetriebe und Start-ups geschaffen werden. Daneben ist langfristig auch die Errichtung von Wohnungen vorgesehen. Nachfolgend wird die Rolle eines Unternehmers herausgearbeitet, der die Idee des Beta-Campus maßgeblich entwickelt hat und eine zentrale Funktion im kleinstädtischen Entscheidungsnetzwerk einnimmt. Entlang der Entwicklungen rund um den Beta-Campus wird sein Beitrag zu sozialräumlichen Wandlungsprozessen diskutiert.

\subsubsection{Lokale Governance-Strukturen und ein Unternehmer als Schlüsselperson}

In Waidhofen/Ybbs spielen Kooperationen zwischen unterschiedlichen Akteursgruppen in verschiedenen Themen- und Planungsfeldern eine zentrale Rolle. Insbesondere zwischen den Akteuren aus Politik und Wirtschaft ist in der Kleinstadt eine enge Abstimmung zu beobachten. Der Bürgermeister genießt insgesamt ein breites Vertrauen bei den Wirtschaftstreibenden im Einzelhandel, den KMUs und den Industriebetrieben (Interview 1, 15 und 16) und setzt sich für die Entwicklung der lokalen Wirtschaft ein. Umkehrt werden in den vergangenen Jahren auch von Unternehmerseite verstärkt Impulse in der Regionalentwicklung gesetzt. Wie eingangs erwähnt, kommt dabei einem Unternehmer aus der Region eine besondere Rolle zu. Er engagiert sich bei verschiedenen Initiativen und Zusammenschlüssen, und nimmt auch bei der Entwicklung des Beta-Campus eine zentrale Bedeutung ein.

Der Unternehmer steht einem regionalen Leitbetrieb mit internationalen Unternehmensstandorten vor und sieht den „Schlüssel zum regionalwirtschaftlichen Erfolg“ in der Attraktivierung der gesamten Region. Von den InterviewpartnerInnen wird er als „strategischer Denker“, „Visionär“ und „Wogenglätter“ charakterisiert, der neben dem notwendigen Fachwissen insbesondere auf sein persönliches Netzwerk zurückgreifen kann (Interview 3 und 4). Da auch sein Unternehmen vom Fachkräftemangel betroffen ist, ist seine Motivation, sich aktiv in die Regionalentwicklung einzubringen, neben regionaler Verbundenheit auch auf persönlichen Handlungsdruck zurückzuführen. Im zeitlichen Rückblick wird deutlich, dass der familiengeführte Leitbetrieb zunächst ein konkurrierendes Verhalten im „Kampf um Fachkräfte“ an den Tag gelegt hat, nun jedoch den Kooperationsgedanken in den Vordergrund stellt (Interview 15).

\subsubsection{Der Unternehmer und die verschiedenen Aspekte sozialräumlichen Wandels}

Nachfolgend wird vor dem Hintergrund der vorgestellten EntrepreneurshipKategorien die Rolle des Unternehmers für die Entstehung des Beta-Campus genauer beleuchtet und die damit verbundenen sozialräumlichen Veränderungen skizziert. 


\section{Erneuerung des regionalen Wirtschaftspfads}

Der Unternehmer kann als Policy Entrepreneur bezeichnet werden, der nicht nur als zentraler Ideengeber des Beta-Campus identifiziert werden kann, sondern auch insgesamt zum inhaltlichen Policy-Wandel in der Region beiträgt. Die metallverarbeitenden produzierenden Betriebe bilden die regionalökonomische Basis der Region und sind ein wichtiger Faktor für die regionale Identität. Mit dem Beta-Campus versucht die Kleinstadt das traditionelle Erbe der Eisenindustrie mit neuen, digitalen Arbeitsformen zu kombinieren. Der Beta-Campus kann als räumliche Manifestierung einer regionalen Pfaderneuerung betrachtet werden. Als Maßnahme gegen Fachkräftemangel und Abwanderung gilt der Beta-Campus als Leuchtturmprojekt, das die Attraktivität der Region als Ganzes steigern soll und die traditionellen Branchen zukunftsfähig machen soll. Dabei wird nicht nur das Problem des Fachkräftemangels adressiert, sondern auch Trends wie Digitalisierung oder Co-Working aufgegriffen und damit neue Arbeitswelten und traditionelle Produktionsweisen zusammengeführt.

\section{Informeller und formeller Institutionenwandel}

Insgesamt haben die Industriebetriebe in Waidhofen/Ybbs und Umgebung als Akteure der Regionalentwicklung in den letzten Jahren an Bedeutung gewonnen. Formell lässt sich dieser Wandel auch anhand der ausgewiesenen Schwerpunkte in der regionalen LEADER-Strategie feststellen. Standen lange Zeit kulturtouristische Projekte in der Region im Vordergrund, rücken mit der aktuellen Förderperiode stärker Maßnahmen für metallverarbeitende Betriebe und junges Unternehmertum in den Fokus der Regionalentwicklung. Diese Entwicklung ist auch auf das aktive Engagement der Stadt Waidhofen/Ybbs und der beschriebenen Schlüsselperson zurückzuführen, die sich insgesamt sehr aktiv in die Erstellung der LEADER-Strategie eingebracht hat und die Interessen der regionalen Industriebetriebe vertreten hat (Interview 2 und 9).

In Waidhofen/Ybbs ist zudem ein informeller Transformationsprozess zu beobachten, der bereits mit Beginn der 2000er Jahren eingesetzt hat. Handelten VertreterInnen der lokalen Wirtschaft und Politik in der Region rückblickend häufig getrennt voneinander, so hat sich dieses Selbstverständnis in den letzten beiden Jahrzehnten gewandelt. Das „kollektive Netzwerkdenken“ wird von nahezu allen InterviewpartnerInnen als Spezifikum der Kleinstadt bzw. der Region angeführt und spielt im Selbstverständnis der Befragten eine große Rolle (u.a. Interview 2, 9, 15). In den Gesprächen wird deutlich, dass dieser Wandel bereits in der Vergangenheit von „Pionieren“ aus Politik und Wirtschaft eingeleitet wurde und durch die heutige „Generation“ von Schlüsselpersonen zunehmend institutionalisiert wird. Der Unternehmer kann somit auch als Institutional Entrepreneur bezeichnet werden, da er nicht nur einen zentralen Akteur im Netzwerk darstellt, sondern das „Netzwerkdenken“ weiterdenkt, aktiv umsetzt und promotet. So wurde unter Mitwirkung des beschriebenen Unternehmers das bestehende „Commitment zur Zusammenarbeit" und gemeinsamen wirtschaftlichen Attraktivierung der Region zwischen den Akteuren aus Politik, Wirtschaft und Regionalentwicklung formalisiert und in Form eines regionalen Leitbilds festgehalten. 


\section{Erweiterung und Formalisierung des Akteursnetzwerks}

Aufgrund der Dimension des Beta-Campus sind zahlreiche öffentliche und private Partner auf verschiedenen Governance-Ebenen in die Projektrealisierung involviert. Wesentlich zum vertikalen und horizontalen Ausbau des regionalwirtschaftlichen Steuerungsgefüges trägt das persönliche Netzwerk des Unternehmers bei. So konnten auch auf nationaler Ebene UnterstützerInnen unterschiedlicher parteipolitischer Zugehörigkeit gewonnen werden, die für das Projekt werben bzw. es finanziell unterstützen. Darüber hinaus werden aktiv lokale Stakeholder verschiedener angrenzender Handlungsbereiche in den Entwicklungsprozess inkludiert. Es erfolgt u.a. eine gezielte Einbindung von lokalen Schulen, um SchülerInnen mit regionalen Unternehmen zusammenzubringen (Interview 5 und 15). Der Beta-Campus kann zudem u.a. an das Akteursnetzwerk einer regionalen Plattform für Bildung und Ausbildung andocken, dem der Unternehmer vorsteht.

Daneben wurde zur künftigen Projektfinanzierung auch der Förderverein BetaCampus bestehend aus rund 40 Unternehmen gegründet, deren Obmann der beschriebene Unternehmer ist (Interview 9). Der informelle Zusammenschluss der Unternehmen, der im Zuge der Beta-Campus Entwicklung entstand, konnte durch die Vereinsgründung als formelles Netzwerk implementiert werden. Die Mitgliedschaft umfasst eine finanzielle Beteiligung der Mitgliedsunternehmen, sieht aber auch besondere Nutzungsrechte des Beta-Campus vor (Interview 5). Ein Blick in die Vergangenheit zeigt, dass der Wettbewerb um Fachkräfte ein verstärktes Konkurrenzverhalten unter den Unternehmen zur Folge hatte. Aufgrund des Engagements von einzelnen Unternehmen und daraus resultierenden verschiedenen Initiativen rückte nach und nach der Kooperationsgedanke in den Vordergrund. Durch die Formalisierung als Förderverein gelingt es den regionalen Unternehmen nun ihre Rolle als zentraler, wenngleich informell tätiger Akteur im Steuerungsgefüge zu verstetigen und die potenziellen Weichen als langfristige Einflussgröße in der Regionalentwicklung zu stellen.

\section{Informelle Interaktionsmodi und Zuständigkeiten}

Zudem haben sich informelle Formen der Interaktion zwischen den zentralen Schlüsselpersonen aus Politik und Wirtschaft rund um den Beta-Campus etabliert. Hervorzuheben ist dabei auch die Bedeutung von kurzen Entscheidungswegen und dem „Sich-Kennen“ im kleinstädtischen Steuerungsgefüge. Im Rahmen von informellen, aber regelmäßigen Treffen werden strategisch die nächsten Schritte in einem „kleinen, feinen Netzwerk“ (Interview 9) aus Politik und Wirtschaft gesetzt, in dem jeder der Beteiligten verschiedene Aufgaben übernimmt, wie ein Akteur aus der Politik berichtet:

„,Wir haben da keine Zuständigkeiten irgendwo definiert, das ergibt sich aus einem immer wieder Zusammentreffen, wir treffen uns wirklich regelmäßig [...]. Das hat eine Eigendynamik bekommen, wo wir das nicht niedergeschrieben haben, sondern wo Leute beisammen sind, die wissen, dass sie dafür verantwortlich sind und sich da auch nicht drücken. "(Interview 9) 


\section{Regionale Erweiterung des Handlungsraums}

Auch die regionale Ausweitung des Handlungsraums (,,re-scaling“) kann ausgehend von Waidhofen/Ybbs als kleinstädtisches Zentrum beobachtet werden. Im Zuge von verschiedenen Projekten und Initiativen, wie u.a. dem Beta-Campus, hat sich in der Region rund um Waidhofen/Ybbs im Bereich der Wirtschaftsentwicklung in den vergangenen Jahren ein Regional Governance-Netzwerk etabliert. So wird auch bei der Entwicklung des Beta-Campus nicht nur bewusst die Zusammenarbeit der Unternehmen in der Region forciert, sondern auch die Ausrichtung des Projekts in einem regionalen Maßstab gedacht. Der Innovations- und Forschungscampus soll künftig als einer von mehreren Standorten mit verschiedenen Schwerpunkten in der Region betrieben werden, die von einer bereits bestehenden regionalen Plattform für Bildung und Forschung koordiniert werden.

\subsection{Diskussion}

Für Waidhofen/Ybbs kann ein umfassender sozialräumlicher Wandlungsprozess festgestellt werden, der sich über die vergangenen zwanzig Jahren erstreckt und neben inhaltlich-politischen und institutionellen Veränderungen auch mit einem Wandel der Governance-Strukturen einhergeht. Insgesamt hat sich die lokale Governance im Bereich Wirtschaft in Waidhofen/Ybbs in den vergangenen Jahren von einem „Nebeneinander“ zu einer „dialogorientierten Vergemeinschaftung“ zwischen Wirtschaft und Politik gewandelt. Diese Vergemeinschaftung basiert auf einer klaren Rollenverteilung zwischen Politik und Wirtschaft: die regionalen Unternehmen nehmen eine aktive, treibende Rolle in diesem Bündnis ein, während die Politik (auch im Selbstverständnis) als unterstützender Part agiert. Zurückzuführen ist dieser Wandel einerseits auf das Engagement von Schlüsselpersonen, andererseits aber auch auf den steigenden Handlungsdruck und gemeinsame Zielsetzungen in Hinblick auf Fachkräftemangel und Abwanderung (Healey 1997).

Die hier vorgestellte Schlüsselperson aus der Wirtschaft zeichnet sich dabei in besonderer Weise durch ihr soziales Kapital und ihre zentrale Position im regionalen Netzwerk aus. Dabei wird deutlich, dass die persönlichen Kontakte des Unternehmers wesentlich zur Umsetzung Zielsetzungen beitragen. Darüber hinaus kann eine enge Verbindung zwischen dem regional verankerten „Netzwerkdenken“ und dem Handeln des Unternehmers festgestellt werden. Einerseits unterstützt dieses regionale Credo den Unternehmer bei seinen Vorhaben, andererseits trägt dieser im Rahmen des BetaCampus zu einer Formalisierung der bestehenden Kooperationen und damit des ,Netzwerkdenkens" bei. In diesem Kontext muss betont werden, dass der vorliegende Beitrag exemplarisch auf die Rolle eines Unternehmers in der Regionalentwicklung fokussiert, in der Vergangenheit und Gegenwart jedoch Schlüsselpersonen aus verschiedenen 
Handlungsbereichen zum sozialräumlichen Wandel in Waidhofen/Ybbs beigetragen haben.

Insgesamt zeigt sich deutlich, dass die zentralen Funktionen im kleinstädtischen Netzwerk von vergleichsweise wenigen Personen ausgeübt werden und dadurch Entscheidungskompetenzen gebündelt werden. Besonders durch die Etablierung von neuen formellen Ämtern und Funktionen können nicht-staatliche Schlüsselpersonen an Macht gewinnen und Prozesse der Regionalentwicklung mitbestimmen. Der Unternehmer verfügt beispielsweise über Schlüsselfunktionen in verschiedenen Organisationen (Vorstand Beta-Campus, regionale Forschungsplattform u.a.) und gewinnt so an Einflussnahme im regionalen Handlungsgefüge. Trotz der Inklusion von Akteuren aus verschiedenen Handlungsfeldern und Ebenen in das Governance-Netzwerk der Kleinstadt (Frisch-Aviram et al. 2018), zeigt sich, dass richtungsgebende Ideen und Impulse zumeist in einem informellen Kleinnetzwerk ausgearbeitet werden, bevor sie einem erweiterten Netzwerk zur formellen Entscheidung vorgelegt werden.

Die Ergebnisse der vorliegenden Fallstudie zeigen, dass das Netzwerk und das Engagement von Schlüsselpersonen sozialräumliche Wandlungsprozesse maßgeblich beeinflussen bzw. beschleunigen können. Gleichzeitig ist es aber auch von institutionellen Rahmenbedingungen, wie der Bereitstellung von finanziellen Fördermitteln, abhängig, ob eine erfolgreiche Umsetzung von Projekten auf lokaler Ebene erfolgt. In diesem Zusammenhang kann eine gemeinsame Zielsetzung und ein kooperatives Ansuchen von Akteuren aus Politik und Wirtschaft um Förderungen um EU-, Bundes- und Landesinstitutionen helfen, den Handlungsspielraum der Kleinstadt auch längerfristig zu sichern (KüHN \& MilSTREY 2015).

\subsection{Ausblick}

Anhand der hier vorgestellten Fallstudie konnte exemplarisch gezeigt werden, was eine Schlüsselperson auszeichnet und inwieweit sie Einfluss auf Prozesse der Regionalentwicklung nehmen kann. Dabei ermöglicht der Fokus auf das Handeln von Einzelpersonen einen Perspektivenwechsel in der Analyse lokaler Governance und erlaubt einen vertieften Einblick in die Mechanismen regionaler Steuerung und damit verbundener sozialräumlicher Entwicklungen. Mittels der konzeptionellen Unterscheidung in Policy, Institutional, und Governance Entrepreneurship konnte eine differenzierte Betrachtung des individuellen Handlungsspielraums in sozialräumlichen Wandlungsprozessen erfolgen. Damit kann die Forschung zu Schlüsselpersonen einen Beitrag zur Beantwortung der Frage leisten, warum sich Regionen trotz vermeintlich vergleichbarer institutioneller Rahmenbedingungen und räumlicher Voraussetzungen unterschiedlich entwickeln. 
Um künftig weiterführende Handlungsempfehlungen für die Praxis der Regionalentwicklung zu formulieren, sind vertiefende vergleichende Fallstudien zu Schlüsselpersonen notwendig. So wäre es z.B. denkbar, aufbauend auf den Forschungserkenntnissen, entsprechende Vernetzungsangebote und Plattformen für engagierte Personen in der Regionalentwicklung zu schaffen oder Experimentierräume zum Austausch und der Entwicklung von Ideen zu etablieren, um innovative Gemeinden und Regionen organisatorisch und finanziell zu unterstützen.

\section{Bibliographie}

Battilana J., Leca B., Boxenbaum E. (2009), 2 How actors change institutions: towards a theory of institutional entrepreneurship. Academy of Management Annals 3, pp. 65-107.

BeER A. (2014), Leadership and the governance of rural communities. Journal of Rural Studies 34, pp. 254-262.

Benz A., Lütz S., Schimank U., Simonis G. (2008), Handbuch Governance: Theoretische Grundlagen und empirische Anwendungsfelder. Wiesbaden, Springer-Verlag.

Bogner A., Menz W. (2009), The theory-generating expert interview: epistemological Interest, forms of knowledge, interaction. In: Bogner A., LitTig B. \& Menz W. (eds.), Interviewing experts. London, Palgrave Macmillan UK.

Christopoulos D., Ingold K. (2011), Distinguishing between political brokerage \& political entrepreneurship. Procedia - Social and Behavioral Sciences 10, pp. 36-42.

Cocks M. (2013), Conceptualizing the role of key individuals in urban governance: cases from the economic regeneration of Liverpool, UK. European Planning Studies 21, pp. 575-595.

Crouch C. (2005), Capitalist diversity and change: recombinant governance and institutional entrepreneurs. Oxford, Oxford University Press.

DöRINGER S. (2020), Individual agency and socio-spatial change in regional development. Conceptualizing governance entrepreneurship. Geography Compass, e12486.

Frisch-Aviram N., Cohen N., BeERI I. (2018), Low-level bureaucrats, local government regimes and policy entrepreneurship. Policy Sciences 51, pp. 39-57.

GAILING L., Ibert O. (2016), Key players: Space as object of and resource in processes of change. Raumforschung und Raumordnung 74, pp. 391-403.

Galanti M.T. (2018), Enablers and Time: How Context Shapes Entrepreneurship in Institutional and Policy Change. In: BAKIR C. \& Jarvis D.S.L. (eds.), Institutional Entrepreneurship and Policy Change: Theoretical and Empirical Explorations. Cham, Springer International Publishing.

Healey P. (1997), Collaborative Planning: Shaping Places in Fragmented Societies. Vancouver, UBC Press.

KIngdon J.W. (1995), Agendas, alternatives, and public policies. New York, Harper Collins.

KüHn M., Milstrey U. (2015), Medium-Sized Cities as Peripheral Centers: Cooperation, Competition and Hierarchy in Shrinking Regions. Raumforschung und Raumordnung 73, pp. 185-202. 
LEICK B., GRETZINGER S. (2018), Brokerage and governance for business networks: a metasynthesisbased discussion. Journal of Management and Governance 22, pp. 773-804.

Mayer H., Baumgartner D. (2014), The role of entrepreneurship and innovation in peripheral regions. disP - The Planning Review 50, pp. 16-23.

Mintrom M., Norman P. (2009), Policy entrepreneurship and policy change. Policy Studies Journal 37, pp. 649-667.

NorTh D. (1990), Institutions, institutional change and economic performance. Cambridge, Cambridge University Press.

Pattberg P. (2017), The emergence of carbon disclosure: Exploring the role of governance entrepreneurs. Environment and Planning C: Politics and Space 35, pp. 1437-1455.

ScharpF F.W. (2000), Interaktionsformen. Akteurzentrierter Institutionalismus in der Politikforschung. Opladen, Leske + Budrich.

SCHUMPETER J.A. (1961), The theory of economic development: an inquiry into profits, capital, credit, interest, and the business cycle (1912/1934). Cambridge, Harvard University Press.

Sotarauta M., Pulkkinen R. (2011), Institutional entrepreneurship for knowledge regions: in search of a fresh set of questions for regional innovation studies. Environment and Planning C: Government and Policy 29, pp. 96-112.

Statistik Austria. (2019), Einwohnerzahl und Komponenten der Bevölkerungsentwicklung Waidhofen an der Ybbs. Blick auf die Gemeide. Wien, Statistik Austria.

WeIK E. (2011), Institutional Entrepreneurship and Agency. Journal for the Theory of Social Behaviour 41, pp. 466-481.

Willi Y., PÜtz M., Mayer H. (2018), Policy entrepreneurship and regional development. CRED Research Papers. University of Bern.

Witzel A. (2000), Das problemzentrierte Interview. Forum Qualitative Sozialforschung / Forum: Qualitative Social Research 1. 